\title{
Searching for higher-dimensional wormholes with noncommutative geometry
}

\author{
Farook Rahaman $^{\text {a }}{ }^{1}$, P.K.F. Kuhfittig ${ }^{\mathrm{b}}$, Saibal Ray ${ }^{\mathrm{c}}$, \\ Safiqul Islam ${ }^{\mathrm{d}}$ \\ ${ }^{a}$ Department of Mathematics, Jadavpur University, Kolkata 700 032, West \\ Bengal, India \\ ${ }^{\mathrm{b}}$ Department of Mathematics, Milwaukee School of Engineering, Milwaukee, \\ Wisconsin 53202-3109, USA \\ ${ }^{\mathrm{c}}$ Department of Physics, Government College of Engineering and Ceramic \\ Technology, Kolkata 700 010, West Bengal, India \\ ${ }^{\mathrm{d}}$ Department of Mathematics, Jadavpur University, Kolkata 700 032, West \\ Bengal, India
}

\begin{abstract}
Noncommutative geometry, an offshoot of string theory, replaces point-like structures with smeared objects and has recently been extended to higher dimensions. The purpose of this paper is to obtain wormhole solutions with this extended noncommutative geometry as a background. It is found through this investigation that wormhole solutions exist in the usual four, as well as in five dimensions, but they do not exist in higher-dimensional spacetimes.
\end{abstract}

\section{Introduction}

The extension of general relativity to higher dimensions was motivated in part by studies of the early Universe. While it is generally believed that in the present Universe the extra spatial dimensions have become compactified, their very existence has led to many investigations in various areas. Rahaman et al. [1] investigated whether the usual solar system tests are compatible with

$\overline{1}$ Corresponding author.

Email addresses: rahaman@iucaa.ernet.in (Farook Rahaman), kuhfitti@msoe.edu (P.K.F. Kuhfittig), saibal@iucaa.ernet.in (Saibal Ray), sofiqul0010yahoo.co.in (Safiqul Islam).

$\begin{array}{ll}\text { Preprint submitted to Elsevier } & 9 \text { October } 2018\end{array}$ 
the existence of higher spatial dimensions. Other studies involved the motion of test particles [2], solar-system effects in a Scharzschild-de Sitter spacetime [3], as well as numerous other phenomena [4,5].

Many studies assume only one extra spatial dimension when discussing higherdimensional cases $[6,7,8,9,10,11,12,13,14]$. In this regard we would like to have a special mention of some of our recent works [13,14]. Ray [13] has considered static spherically symmetric charged dust corresponding to $(n+2)$ dimensional Einstein-Maxwell spacetime and shown that for $n=3$, the expression for gravitational mass corresponds to the mass given by Bonnor [15] and Cohen and Cohen [16] which, in turn, confirms the identification of mass as the fifth dimension by Ponce de Leon [17]. In the later work, Kuhfittig [14] assumes that the 3-brane is a de Sitter space and there is only one extra spatial dimension, assumed to be time dependent. It is proposed in this work that the cosmological inflation of the 3-brane may provide a possible explanation for the collapse of the extra dimension, as well as for the energy stored in the resulting curled-up dimension.

An important outcome of string theory (which assumes extra dimensions) is the notion that coordinates may become noncommutative operators in a $D$ brane $[18,19]$. The consequence is a fundamental discretization of spacetime due to the commutator $\left[\mathbf{x}^{\mu}, \mathbf{x}^{\nu}\right]=i \theta^{\mu \nu}$, where $\theta^{\mu \nu}$ is an antisymmetric matrix, similar to the way that the Planck constant $\hbar$ discretizes phase space [20]. This noncommutative geometry is an intrinsic property of spacetime that does not depend on particular features such as curvature. Moreover, it was pointed out in Ref. [21] that noncommutativity replaces point-like structures by smeared objects, thereby eliminating the divergences that normally appear in general relativity. This smearing can be modeled by the use of the Gaussian distribution of minimal length $\sqrt{\theta}$ instead of the Dirac delta function. So the energy density of the static and spherically symmetric smeared and particle-like gravitational source has the form [22]

$$
\rho(r)=\frac{M}{(4 \pi \theta)^{3 / 2}} e^{-r^{2} / 4 \theta} .
$$

The mass $M$ could be a diffused centralized object such as a wormhole [23]. The Gaussian source has also been used by Sushkov [24] to model phantomenergy supported wormholes, as well as by Nicolini and Spalluci [25] for the purpose of modelling the physical effects of short-distance fluctuations of noncommutative coordinates in the study of black holes. Galactic rotation curves inspired by a noncommutative-geometry background are discussed in one of our very recent works [26]. The stability of a particular class of thin-shell wormholes in noncommutative geometry is analyzed elsewhere [27].

Therefore, the purpose of this paper is to obtain wormhole solutions within 
the framework of noncommutative geometry, first by assuming the usual four dimensions in general relativity and then by adding one extra spatial dimension. It was subsequently discovered, however, that for dimensions above five, no wormhole solutions exist, pointing out the danger of assuming only one extra spatial dimension.

\section{Basic Equations}

To describe a spherically symmetric wormhole spacetime in higher dimension, we take the metric to be

$$
d s^{2}=-e^{\nu(r)} d t^{2}+e^{\lambda(r)} d r^{2}+r^{2} d \Omega_{n}^{2}
$$

where the line element $d \Omega_{n}{ }^{2}$ on the unit $n$-sphere is given by

$$
d \Omega_{n}{ }^{2}=d \theta_{1}{ }^{2}+\sin ^{2} \theta_{1} d \theta_{2}{ }^{2}+\sin ^{2} \theta_{1} \sin ^{2} \theta_{2} d \theta_{3}{ }^{2}+\ldots \ldots \ldots \ldots \ldots+\prod_{i=1}^{n-1} \sin ^{2} \theta_{i} d \theta_{n}(3)
$$

The most general energy momentum tensor compatible with static spherically symmetry is

$$
T_{\nu}^{\mu}=\left(\rho+p_{r}\right) u^{\mu} u_{\nu}-p_{r} g_{\nu}^{\mu}+\left(p_{t}-p_{r}\right) \eta^{\mu} \eta_{\nu}
$$

with $u^{\mu} u_{\mu}=-\eta^{\mu} \eta_{\mu}=1$.

The Einstein equations are

$$
\begin{aligned}
& e^{-\lambda}\left(\frac{n \lambda^{\prime}}{2 r}-\frac{n(n-1)}{2 r^{2}}\right)+\frac{n(n-1)}{2 r^{2}}=8 \pi \rho, \\
& e^{-\lambda}\left(\frac{n(n-1)}{2 r^{2}}+\frac{n \nu^{\prime}}{2 r}\right)-\frac{n(n-1)}{2 r^{2}}=8 \pi p_{r}, \\
& \frac{1}{2} e^{-\lambda}\left[\frac{1}{2}\left(\nu^{\prime}\right)^{2}+\nu^{\prime \prime}-\frac{1}{2} \lambda^{\prime} \nu^{\prime}+\frac{(n-1)}{r}\left(\nu^{\prime}-\lambda^{\prime}\right)+\frac{(n-1)(n-2)}{r^{2}}\right] \\
& \quad-\frac{(n-1)(n-2)}{2 r^{2}}=8 \pi p_{t} .
\end{aligned}
$$


In higher dimensions, the energy density of the static and spherically symmetric smeared and particle-like gravitational source having a minimal spread Gaussian profile is taken as [28]

$$
\rho=\frac{M}{(4 \pi \theta)^{(n+1) / 2}} \exp \left(-\frac{r^{2}}{4 \theta}\right),
$$

where $M$ is the total mass of the source which is diffused throughout a region of linear dimension $\sqrt{\theta}$ due to the uncertainty.

\section{Solutions}

We are going to assume a constant redshift function for our model, the socalled zero-tidal force solution [29], to make the wormhole traversable by humanoid travelers. In other words, we have

$$
\nu=\nu_{0},
$$

where $\nu_{0}$ is a constant.

Using Eq. (9), we can rewrite the field equations (5)-(7) in terms of the shape function $b(r)$, where $b(r)=r\left(1-e^{-\lambda}\right)$ :

$$
\begin{aligned}
& \frac{n b^{\prime}}{2 r^{2}}+\frac{n(n-2) b}{2 r^{3}}=\frac{8 \pi M}{(4 \pi \theta)^{\frac{n+1}{2}}} \exp \left(-\frac{r^{2}}{4 \theta}\right), \\
& 8 \pi p_{r}=-\frac{n(n-1) b}{2 r^{3}} \\
& 8 \pi p_{t}=\frac{(3-n)(n-1) b}{2 r^{3}}-\frac{(n-1) b^{\prime}}{2 r^{2}} .
\end{aligned}
$$

From Eq. (10), we get the following solution for the shape function:

$b(r)=\frac{16 \pi}{n r^{n-2}(4 \pi \theta)^{(n+1) / 2}} \frac{\left[2^{n}\left(\frac{1}{\theta}\right)^{-(n+1) / 2}\left\{r^{n+1} 2^{(1-n) / 2}\left(\frac{1}{\theta}\right)^{(n+1) / 2}\left(\frac{r^{2}}{\theta}\right)^{-(n+1) / 4} \exp \left(-\frac{r^{2}}{8 \theta}\right) M_{W}\left(\frac{n+1}{4}, \frac{n+3}{4}, \frac{r^{2}}{4 \theta}\right)\right\}\right]}{(n+3)\left(\frac{n+1}{2}\right)}$
$+\frac{16 \pi}{n r^{n-2}(4 \pi \theta)^{(n+1) / 2}} \frac{\left[2^{(3-n) / 2} r^{n-1}\left(\frac{1}{\theta}\right)^{(n-1) / 2}\left(\frac{r^{2}}{\theta}\right)^{-(n+1) / 4} \exp \left(-\frac{r^{2}}{8 \theta}\right) M_{W}\left(\frac{n+5}{4}, \frac{n+3}{4}, \frac{r^{2}}{4 \theta}\right)\right]}{\frac{n+1}{2}}+\frac{C}{r^{n-2}}$ 
where $C$ is an integration constant and the Whittaker mass $M_{W}$ can be defined as

$$
M_{W}(\mu, \nu, z)=\exp \left(-\frac{z}{2}\right) z^{(1 / 2+\nu)} \text { hypergeom }\left(\left[\frac{1}{2}+\nu-\mu\right],[1+2 \nu], z() 14\right)
$$

This solution is obviously very difficult to deal with. In the following subsections we will therefore discuss various cases for specific dimensions. However, $C$ being an integration constant, mathematically, $b(r)$ is a solution for every values of $C$. The flare-out condition, on the other hand, is a physical requirement that is satisfied only for a certain range of values of $C$, which, in turn, depend on the other parameters. Because of the complexity of the shape function, we will use a graphical approach to analyze the wormhole structure, including the location of the throat, in terms of typical values of the parameters. In this manner it is shown that wormhole solutions exist in four and five dimensions only. The higher-dimensional cases require some additional discussion and will be dealt with in the later phase.

\section{$3.1 n=2$}

When $n=2$, i.e., for a four-dimensional spacetime, the shape function of the wormmhole takes the following form:

$$
b(r)=\frac{M}{\sqrt{\pi \theta^{3}}}\left[-2 \theta r \exp \left(-\frac{r^{2}}{4 \theta}\right)+2 \theta^{\frac{3}{2}} \sqrt{\pi} \operatorname{erf}\left(\frac{r}{2 \sqrt{\theta}}\right)+C\right] .
$$

The other parameters are

$$
8 \pi p_{r}=-\frac{1}{r^{3}}\left[\frac{M}{\sqrt{\pi \theta^{3}}}\left\{-2 \theta r \exp \left(-\frac{r^{2}}{4 \theta}\right)+2 \theta^{\frac{3}{2}} \sqrt{\pi} \operatorname{erf}\left(\frac{r}{2 \sqrt{\theta}}\right)+C\right\}\right]
$$

and

$$
\begin{array}{r}
8 \pi p_{t}=\frac{1}{2 r^{3}}\left[\frac{M}{\sqrt{\pi \theta^{3}}}\left\{-2 \theta r \exp \left(-\frac{r^{2}}{4 \theta}\right)+2 \theta^{\frac{3}{2}} \sqrt{\pi} \operatorname{erf}\left(\frac{r}{2 \sqrt{\theta}}\right)+C\right\}\right] \\
-\frac{8 \pi M}{(4 \pi \theta)^{3 / 2}} \exp \left(-\frac{r^{2}}{4 \theta}\right) \cdot(17
\end{array}
$$

The next step is to verify that the shape function has all the properties required for a wormhole structure. To do so, we need to assign some typical values to 


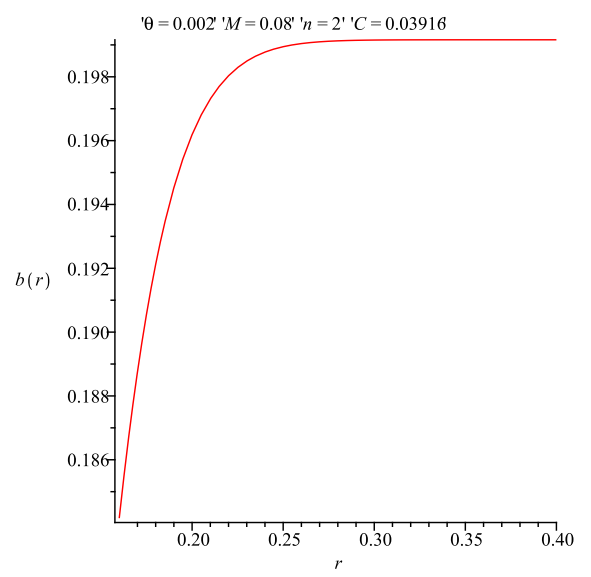

Fig. 1. Diagram of the shape function of the wormhole in four dimension for the specific values of the parameters as $\theta=0.002, M=0.08$ and $C=0.03916$.

the parameters, an example of which is shown in Fig. 1. We can see from Fig. 2 that $b(r) / r \rightarrow 0$ as $r \rightarrow \infty$, so that in conjunction with the constant redshift function, the spacetime is asymptotically flat. The throat of the wormhole is located at $r=r_{0}$, where $G(r)=b(r)-r$ cuts the $r$-axis, shown in Fig. 3 .

Fig. 3 also indicates that for $r>r_{0}, G(r)<0$, i.e., $b(r)-r<0$, which implies that $b(r) / r<1$ for $r>r_{0}$, an essential requirement for a shape function. Moreover, $G(r)$ is a decreasing function for $r \geq r_{0}$. Since $G^{\prime}(r)<0$, we have $b^{\prime}\left(r_{0}\right)<1$, which is the flare-out condition. With this condition now satisfied, the shape function has produced the desired wormhole structure. For completeness let us note that for the given parameters, $r_{0}=0.1955$ to four decimal places with $b^{\prime}(0.1955) \approx 0.20$, obtained from Eq. (10).

The close connection between the flare-out condition and the energy conditions calls for a check on the latter. According to Fig. 4, the null energy condition (NEC) is satisfied, but the weak energy condition (WEC) and strong energy condition (SEC) are violated.

\section{2 $n=3$}

When $n=3$, i.e., for a five-dimensional spacetime, the shape function of the wormmhole is given by

$$
b(r)=\left[\frac{-2 \theta\left(4 \theta+r^{2}\right) M \exp \left(-\frac{r^{2}}{4 \theta}\right)}{3 \pi r \theta^{2}}\right]+\frac{C}{r} .
$$




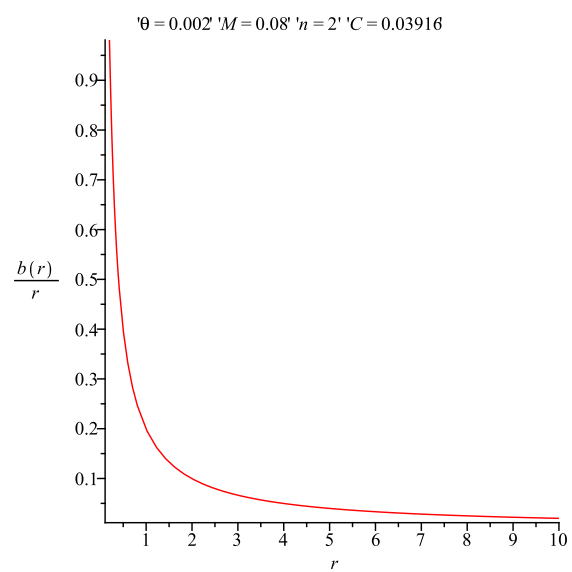

Fig. 2. Asymptotic behavior of the shape function of the wormhole given in Fig.1.

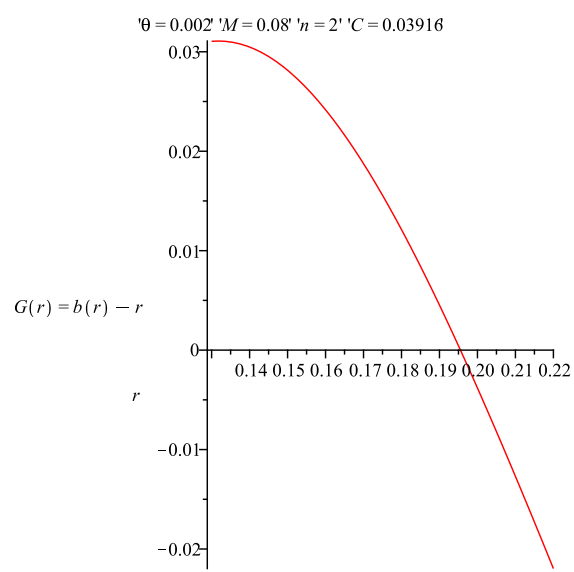

Fig. 3. The throat of the wormhole given in Fig.1, occurs where $G(r)$ cuts the $r$-axis.

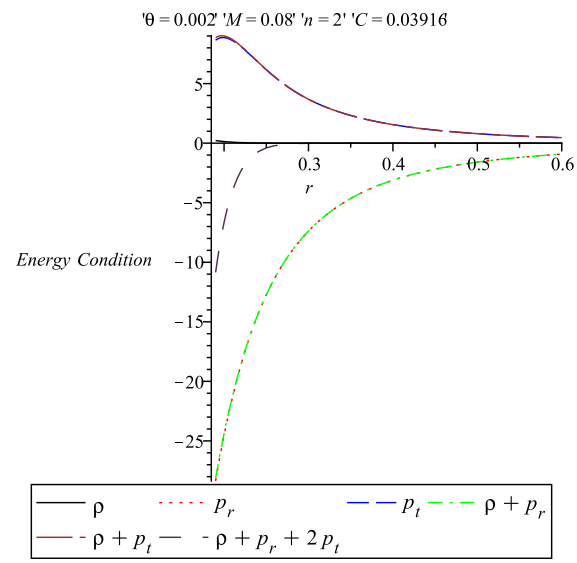

Fig. 4. The variations of the left-hand sides of the expressions for the energy conditions of the matters comprising the four dimensional wormhole are plotted against $r$. 
The other parameters are

$$
8 \pi p_{r}=-\frac{3}{r^{3}}\left\{\left[\frac{-2 \theta\left(4 \theta+r^{2}\right) M \exp \left(-\frac{r^{2}}{4 \theta}\right)}{3 \pi r \theta^{2}}\right]+\frac{C}{r}\right\}
$$

and

$$
\begin{array}{r}
8 \pi p_{t}=\frac{1}{r^{3}}\left\{\left[\frac{-2 \theta\left(4 \theta+r^{2}\right) M \exp \left(-\frac{r^{2}}{4 \theta}\right)}{3 \pi r \theta^{2}}\right]+\frac{C}{r}\right\} \\
-\frac{16 \pi M}{3(4 \pi \theta)^{2}} \exp \left(-\frac{r^{2}}{4 \theta}\right) .
\end{array}
$$

One can see from Fig. 5 that $b(r)=r$ at some point. More precisely, for the particular parameters chosen, $b(0.0878)=0.0878$ to four decimal places. This is in agreement with Fig. 6, which shows $G(r)$ intersecting the $r$-axis at $r=r_{0}$. Also, from Eq. (10), $b^{\prime}(0.0878) \approx 0.34<1$. The function continues to increase to the right of $r_{0}=0.0878$; its slope and is still positive at $r_{1}=0.0893$ but soon becomes negative. So we have a valid solution around the throat. Moreover, $r_{1}=0.0893$ is a convenient cut-off for the wormhole material and subsequent junction to the corresponding external Schwarzschild spacetime

$$
d s^{2}=-\left(1-\frac{2 \mu}{r^{2}}\right) d t^{2}+\left(1-\frac{2 \mu}{r^{2}}\right)^{-1} d r^{2}+r^{2}\left(d \theta_{1}^{2}+\sin ^{2} \theta_{1} d \theta_{2}^{2}+\sin ^{2} \theta_{1} \sin ^{2} \theta_{2} d \theta_{3}^{2}\right) .
$$

Here $\mu$ is related to the mass ' $m$ ' of the five dimensional Schwarzschild black hole as $\mu=\frac{4 G m}{3 \pi}$. Therefore, for the present case it can be evaluated as $\mu=$ $\frac{1}{2} r_{1} b\left(r_{1}\right) \approx 0.0039318$, while $e^{\nu}=\left(1-\frac{2 \mu}{r_{1}^{2}}\right)$, so that $\nu \approx \ln \left(1-\frac{0.0078636}{r_{1}^{2}}\right) \approx$ -4.27546 . This yields the respective interior and exterior line elements:

$$
d s^{2}=-e^{-4.27546} d t^{2}+\frac{d r^{2}}{\left(1-\frac{b(r)}{r}\right)}+r^{2}\left(d \theta_{1}^{2}+\sin ^{2} \theta_{1} d \theta_{2}^{2}+\sin ^{2} \theta_{1} \sin ^{2} \theta_{2} d \theta_{3}^{2}\right)
$$

for $r<r_{1}$ and

$$
\begin{aligned}
d s^{2} & =-\left(1-\frac{0.0078636}{r^{2}}\right) d t^{2}+\frac{d r^{2}}{\left(1-\frac{0.0078636}{r^{2}}\right)} \\
& +r^{2}\left(d \theta_{1}^{2}+\sin ^{2} \theta_{1} d \theta_{2}^{2}+\sin ^{2} \theta_{1} \sin ^{2} \theta_{2} d \theta_{3}^{2}\right)
\end{aligned}
$$

for $r \geq r_{1}$. (Due to the spherical symmetry, the remaining components are already continuous [30]). Observe that only the interior solution retains the 


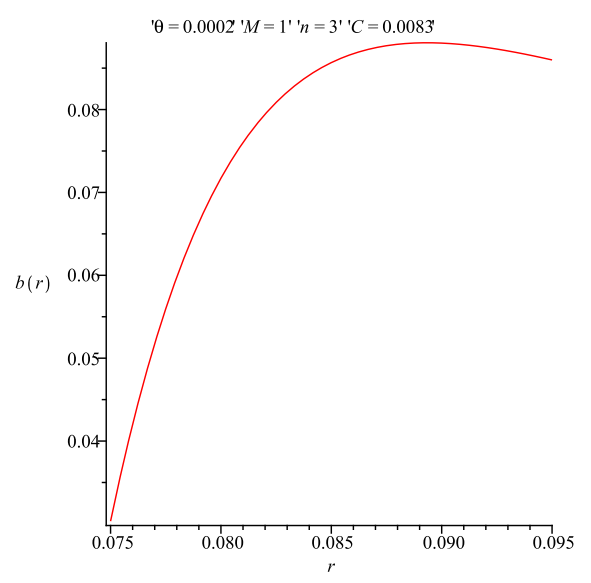

Fig. 5. Diagram of the shape function of the wormhole in five dimension for the specific values of the parameters as $\theta=0.0002, M=1$ and $C=0.0083$. .

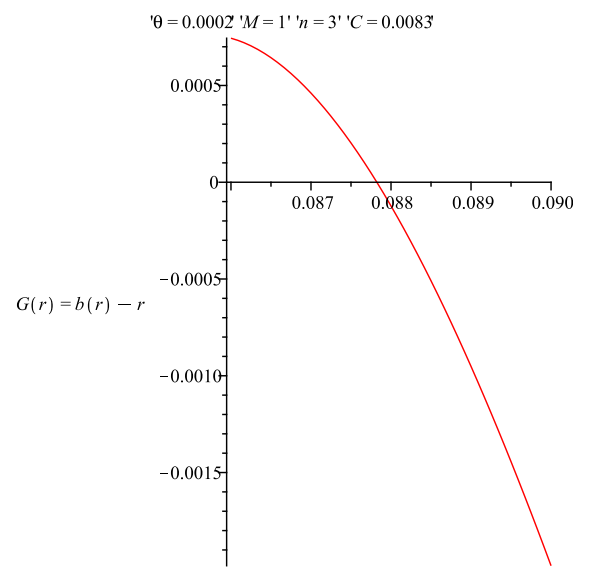

Fig. 6. The throat of the wormhole given in Fig.5, occurs where $G(r)$ cuts the $r$-axis.

zero tidal forces. One can note that here the junction is a thin shell. Since the shell is infinitely thin, the radial pressure is zero. According to the DarmoisIsrael junction condition [31], the surface density is also zero, however, the tangential pressure is nonzero (see Ref. [32] for details).

For the five dimensional case, Fig. 7 indicates that only the NEC is satisfied, while the WEC and SEC are violated.

\section{$3.3 n=4$ and $n=5$}

When $n=4$, i.e., for a six-dimensional spacetime, the shape function of the wormmhole is 


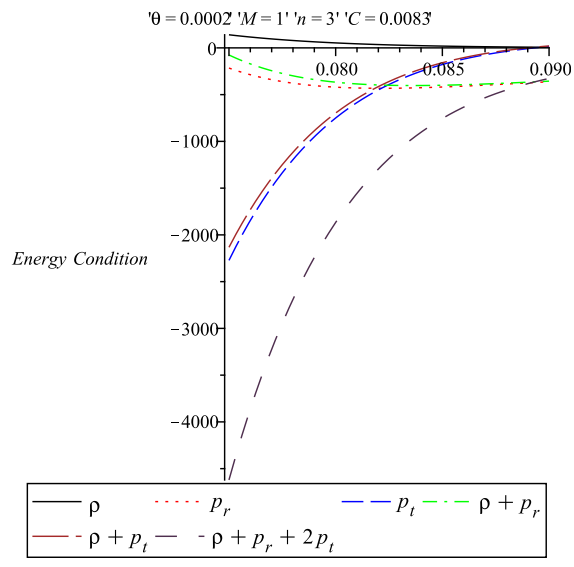

Fig. 7. The variations of left-hand sides of the expressions for the energy conditions of the matters comprising the five dimensional wormhole are plotted against $r$.

$$
\begin{aligned}
b(r) & =\frac{M}{8 \pi r^{2} \sqrt{\pi \theta^{5}}}\left[-2 r^{3} \theta \exp \left(-\frac{r^{2}}{4 \theta}\right)+6 \theta\left\{-2 \theta r \exp \left(-\frac{r^{2}}{4 \theta}\right)+2 \theta^{\frac{3}{2}} \sqrt{\pi} \operatorname{erf}\left(\frac{r}{2 \sqrt{\theta}}\right)\right\}\right] \\
& +\frac{C}{r^{2}} .
\end{aligned}
$$

The other parameters are

$$
\begin{aligned}
8 \pi p_{r} & =-\frac{6}{r^{3}}\left[\frac{M}{8 \pi r^{2} \sqrt{\pi \theta^{5}}}\left\{-2 r^{3} \theta \exp \left(-\frac{r^{2}}{4 \theta}\right)+6 \theta\left(-2 \theta r \exp \left(-\frac{r^{2}}{4 \theta}\right)+2 \theta^{\frac{3}{2}} \sqrt{\pi} \operatorname{erf}\left(\frac{r}{2 \sqrt{\theta}}\right)\right)\right\}\right] \\
& -\frac{6 C}{r^{5}} \\
8 \pi p_{t} & =\frac{3 M}{16 \pi r^{5} \sqrt{\pi \theta^{5}}}\left[-2 r^{3} \theta \exp \left(-\frac{r^{2}}{4 \theta}\right)+6 \theta\left\{-2 \theta r \exp \left(-\frac{r^{2}}{4 \theta}\right)+2 \theta^{\frac{3}{2}} \sqrt{\pi} \operatorname{erf}\left(\frac{r}{2 \sqrt{\theta}}\right)\right\}\right] \\
& +\frac{3 C}{2 r^{5}}-\frac{6 \pi M}{(4 \pi \theta)^{\frac{5}{2}}} \exp \left(-\frac{r^{2}}{4 \theta}\right)
\end{aligned}
$$

Fig. 9 locates the presumptive throat of the wormhole and Fig. 8 shows the shape function $b(r)$. Observe that $b(r)$ is strictly decreasing, a problem that also occurs in the case $n=5$ i.e. for a seven dimensional spacetimes (see Fig. $10)$. For $n=5$, we get

$$
b(r)=\frac{1}{r^{3}}\left[C-\frac{\left(32 \theta^{2}+8 \theta r^{2}+r^{4}\right) M \exp \left(-\frac{r^{2}}{4 \theta}\right)}{10 \pi^{2} \theta^{2}}\right]
$$

So it appears that there are no wormhole solutions for dimensions above five as $b(r)$ contains the factor $\frac{1}{r^{n-2}}(n \geq 2)$ which causes $b(r)$ to be a monotonic 


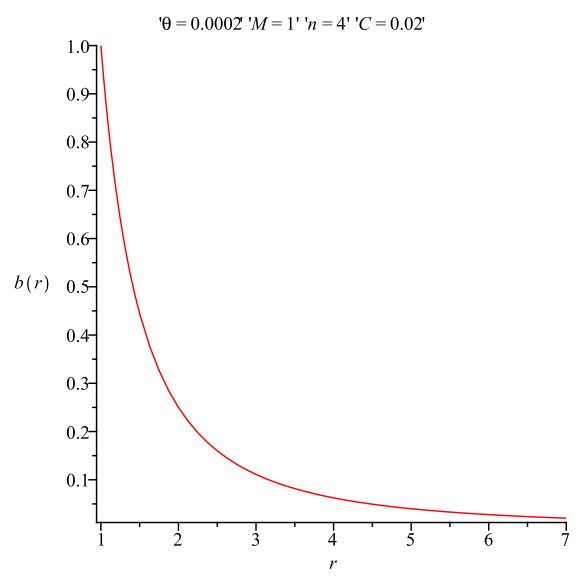

Fig. 8. Diagram of the presumptive shape function of the wormhole in six dimension for the specific values of the parameters as $\theta=0.0002, M=1$ and $C=0.02$.

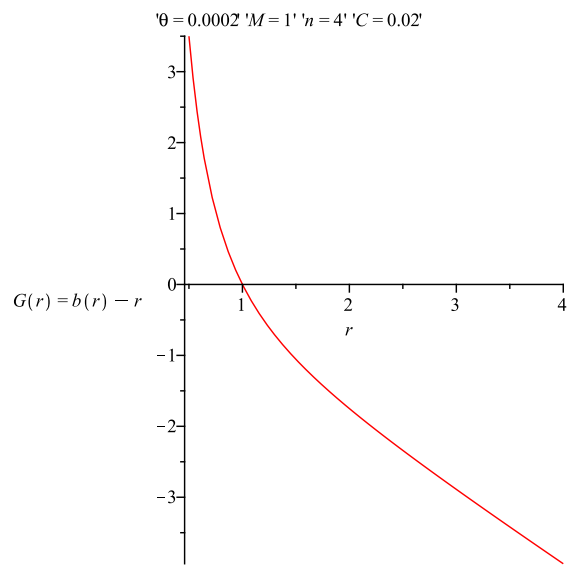

Fig. 9. The throat of the wormhole given in Fig. 8 normally occurs where $G(r)$ cuts the $r$-axis.

decreasing function. The reason is that both $\exp \left(-\frac{r^{2}}{4 \theta}\right)$ and $\operatorname{erf}\left(\frac{r}{2 \sqrt{\theta}}\right)$ level off rapidly in the outward radial direction. As a result, $b^{\prime}(r)$ is completely dominated by $\left(\frac{d}{d r}\right)\left(\frac{C}{r^{n-2}}\right)$ for larger $n$ and proper choice of $C$. So one would expect the slope of $b(r)$ to become even steeper with increasing $n$, which is clearly born out in Figs. 8 and 10. The steepness can only increase further as $n$ increases. For smaller $n$, this dominance does not necessarily hold. Thus, according to Fig. 5 , for $n=3$ and proper choice of $C$, the term $\frac{C}{r}$ allows $b(r)$ to increase long enough to yield an interior solution. For the case $n=2$, none of these arguments apply, and the result is an asymptotically flat spacetime. 


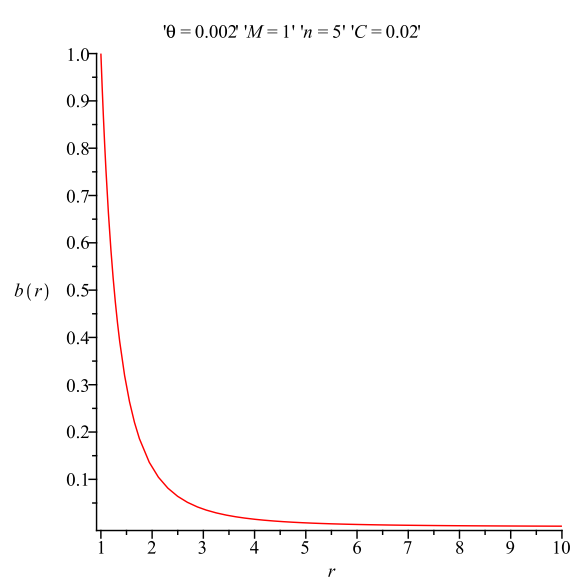

Fig. 10. Diagram of the presumptive shape function of the wormhole in seven dimension for the specific values of the parameters as $\theta=0.0002, M=1$ and $C=0.02$.

\section{Conclusion}

Noncommutative geometry, an offshoot of string theory, replaces point-like structures by smeared objects and has recently been extended to higher dimensions. In this paper we obtain two wormhole solutions within the framework of this extended noncommutative geometry. In general, the primary ingredient for sustaining traversable wormholes is the presence of exotic matter that violates the null energy condition. However, we have proposed a set of new wormhole solutions within the framework of noncommutative geometry background, where the matter satisfies the null energy condition (NEC) but violates the weak energy condition (WEC) and strong energy condition (SEC). It is shown that wormhole solutions exists in the usual four, as well as in five dimensions, but they do not exist in six or seven dimensions, suggesting that such solutions do not exist beyond five dimensions. This outcome points out the danger of assuming only one extra spatial dimension, as is often done. In the analysis of this work, we considered a constant redshift function, the so-called zero-tidal force solution to make the wormhole traversable by humanoid travelers. This assumption simplified the mathematical calculations, but it also provided sufficiently exciting exact solutions. It is worthwhile to mention here that the wormhole in four dimensional spacetime is asymptotically flat whereas in five dimension it is asymptotically non-flat and for higher than five dimensions no wormhole exists. Thus up to four dimension, one can get a regular wormhole and for five dimensional spacetime, one gets wormhole geometry only in a very restricted region. The possibility of getting a wormhole geometry will cease beyond five dimensions. 


\section{Acknowledgments}

FR and SR are thankful to the Inter University Centre for Astronomy and Astrophysics (IUCAA), India for providing research facilities. FR is also grateful to UGC, India for financial support under its Research Award Scheme. We would like to thank the referee for his useful comments to improve substantially the present work.

\section{References}

[1] F. Rahaman, S. Ray, M. Kalam and M. Sarker, Int. J. Theor. Phys. 48, 3124 (2009).

[2] H. Liu and J.M. Overduin, Astrophys. J. 538, 386 (2000).

[3] V. Kagramanova, J. Kunz and C. L. Lämmerzahl, Phys. Lett. B 634465 (2006).

[4] L. Iorio, J. Cosmol. Astropart. Phys. 9, 6 (2005).

[5] L. Iorio, Nat. Sci. 2, 329 (2010).

[6] P.S. Wesson, Astron. Astrophys. 119, 145 (1983).

[7] T. Fukui, Gen. Relativ. Gravit. 19, 43 (1987).

[8] A. Banerjee, B. K. Bhui and S. Chatterjee, Astron. Astrophys. 232, 305, (1990).

[9] S. Chatterjee and B.K. Bhui, Astrophys. Space Sci. 167, 61 (1990).

[10] L. Randall and R. Sundrum, Phys. Rev. Lett. 83, 3370 (1999).

[11] L. Randall and R. Sundrum, Phys. Rev. Lett. 83, 4690 (1999).

[12] J. Ponce de Leon, Gen. Relativ. Gravit. 35, 1365 (2003).

[13] S. Ray, Int. J. Mod. Phys. D 15, 917 (2006)

[14] P.K.F. Kuhfittig, Int. J. Pure Appl. Math. 49, 577 (2008).

[15] W.B. Bonnor, Z. Phys. 160, 59 (1960).

[16] J.M. Cohen and M.D. Cohen, Nuo. Cim. B 60, 241 (1969).

[17] J. Ponce de Leon, Gen. Relativ. Gravit. 35, 1365 (2003).

[18] E. Witten, Nucl. Phys. B 460, 335 (1996).

[19] N. Seiberg and E. Witten, J. High Ener. Phys. 9909, 032 (1999).

[20] A. Gruppuso, J. Phys. A 38, 2039 (2005). 
[21] A. Smailagic and E. Spalluci, J. Phys. A 36, L467 (2003).

[22] P. Nicolini, A Smailagic and E. Spalluci, Phys. Lett. B 632, 547 (2006).

[23] F.S.N. Lobo and R. Garattini, arXiv: 1004.2520.

[24] S.V. Sushkov, Phys. Rev. D 71, 043520 (2005).

[25] P. Nicolini and E. Spalluci, Class. Quantum Gravit. 27, 015010 (2010).

[26] F. Rahaman, P.K.F. Kuhfittig, K. Chakraborty, A.A. Usmani and S. Ray, Gen. Relativ. Gravit. 44, 905 (2012).

[27] P.K.F. Kuhfittig, Adv. High Ener. Phys. 2012, 462493 (2012).

[28] E. Spallucci, A. Smailagic and P. Nicolini, Phys. Lett. B 670, 449 (2009).

[29] M.S. Morris and K.S. Thorne, Am. J. Phys. 56, 395 (1988).

[30] J.P.S. Lemos, F.S.N. Lobo and S. Quinet de Oliveira, Phys. Rev. D 68, 064004 (2003).

[31] W. Israel, Nuo. Cim. B 44, (1966) 1; erratum - ibid. 48, (1967) 463.

[32] F. Rahaman, M. Kalam and S. Chakraborty, Gen. Relativ. Gravit. 38, (2006) 1687. 